\title{
Herbivore associated elicitor-induced defences are highly specific among closely related Nicotiana species
}

\author{
Shuqing $\mathrm{Xu}^{*}$, Wenwu Zhou, Sarah Pottinger and lan T Baldwin
}

\begin{abstract}
Background: Herbivore-induced defence responses are often specific - different herbivores induce different defence responses in plants - and their specificity is largely mediated by chemical cues (herbivore-associated elicitors: HAEs) in insect oral or oviposition secretions. However, the specificity and the mechanisms of HAE-induced defence have not been investigated in the context of the evolutionary relationships among plant species. Here we compare the responses of six closely related Nicotiana species to a synthetic elicitor, N-linolenoyl-glutamic acid (C18:3-Glu) and HAE of two insect herbivores (the Solanaceae specialist Manduca sexta and generalist Spodoptera littoralis).
\end{abstract}

Results: HAE-induced defences are highly specific among closely related Nicotiana species at three perspectives. 1) A single Nicotiana species can elicit distinct responses to different HAEs. N. pauciflora elicited increased levels of JA and trypsin proteinase inhibitors (TPI) in response to C18:3-Glu and the oral secretions of M. sexta (OS Ms) but not to oral secretions of $\mathrm{S}$. littoralis $\left(\mathrm{OS}_{\left.S_{\mathrm{S}}\right)}\right.$. In contrast, N. miersii only responded to $\mathrm{OS}_{S_{S}}$ but not to the other two HAEs. The specific responses to different HAEs in Nicotiana species are likely due to the perception by the plant of each specific component of the HAE. 2) One HAE can induce different defence responses among closely related Nicotiana species. OS Ms $_{\text {and }}$ C18:3-Glu induced JA and TPI accumulations in N. linearis, N. attenuata, N. acuminata and N. pauciflora, but not in N. miersii and N. obtusifolia. 3) The effect of HAE-induced defences differ for the Solanaceae specialist M. sexta and the generalist S. littoralis. Among the four tested Nicotiana species, while the growth rate of $M$. sexta was only reduced by the induced defences elicited by C18:3-Glu; the growth rate of S. littoralis can be reduced by the induced defences elicited by all three HAEs. This is likely due to differences in the susceptibility of the specialist M. sexta and generalist $S$. littoralis to induced defences of their host.

Conclusions: Closely related Nicotiana species elicit highly specific defence responses to herbivore associated elicitors and provide an ideal framework for investigating the molecular mechanisms and evolutionary divergence of induced resistance in plants.

Keywords: Specificity of herbivore induced defence, Nicotiana, Jasmonic acid, Trypsin proteinase inhibitor, Induced resistance, Specialist and generalist

\section{Background}

Induced defences are widespread in plants and play an important role for plant fitness [1]. In response to herbivore attack, plants distinguish mechanical damage from damage caused by feeding insects through the perception of chemical cues (herbivore-associated elicitors: HAEs) in insect oral secretions (OS) [2]. Such HAE-induced plant

\footnotetext{
* Correspondence: sxu@ice.mpg.de

Department of Molecular Ecology, Max Planck Institute for Chemical Ecology, Hans-Knöll-Straße 8, D-07745 Jena, Germany
}

defences have frequently been shown to be insect speciesspecific [3-6]. This is because elicitors from different herbivore species vary both qualitatively and quantitatively and the ability to respond to HAEs varies among plant species $[4,6]$.

The specificity of HAE-induced plant responses appear at multiple levels. The phytohormone jasmonic acid (JA) and its derivatives play a central role in the activation of defences against most insect herbivores $[7,8]$. The induced accumulation of JA in plants can be HAE-specific. For example, in eggplant two fatty acid amino acid conjugates 
(FACs), volicitin and $N$-linolenoyl-Gln, induced more than a two-fold increase in JA levels in comparison with wounding alone. However two other HAEs tested, caeliferin A16:0 and inceptin, did not induce JA accumulation [6]. This suggests that the specificity of HAE-induced JA accumulation is probably mediated by specific receptorligand interactions, although the molecular mechanisms remain to be understood. The specificity of induced JA accumulation among different HAEs can also be mediated by hormonal crosstalk $[4,9,10]$. In $N$. attenuata, Spodoptera exigua oral secretion $\left(\mathrm{OS}_{S e}\right)$ induced lower levels of JA accumulation than Manduca sexta oral secretion $\left(\mathrm{OS}_{M s}\right)$. This is due to enhanced glucose oxidase (GOX) activity in S. exigua OS eliciting a salicylic acid (SA) burst which attenuates JA induction [11]. Furthermore, different plant species can display JA responses with a different timing and/or magnitude after exposure to the same HAE. While volicitin induces JA accumulations in maize, eggplant and soybean, this elicitor does not induce JA accumulations in Arabidopsis thaliana and cowpea [6]. This indicates that the HAE-induced JA response varies among different plant families. However, the variation of such a response among closely related species and the extent to which its specificity is mediated by receptor-ligand interaction [12] or hormonal cross-talks is largely unknown.

In addition to promoting phytohormone accumulation, HAE stimulation can result in accumulation and mobilisation of defence compounds, such as trypsin proteinase inhibitors (TPI) and diterpene glycosides (DTG) which function as direct defences [13,14]. Because most HAEinduced metabolomic responses are thought to be mediated by JA $[13,15,16]$, the specificity of the HAE-induced response is thought to be largely associated with the specificity of HAE-induced JA accumulations. However, other HAE-induced phytohormones can also fine-tune induced metabolomic responses [17].

Certain induced plant defences can reduce the growth and fecundity of insect herbivores, but the effect of an induced defence on insect growth varies among insect species $[18,19]$. As a generalisation, specialist herbivores tend to have a higher tolerance for the toxins produced by their host plant than do generalist herbivores [20]. Therefore, specialist herbivores are on average less sensitive to the changes of their host plant defences than generalists are [20]. Thus it is thought that the induced resistance to a specialist herbivore is more specific (elicited by only a few specific HAEs) than the induced resistance to a more generalist herbivore (elicited by larger number of different HAEs). However, this hypothesis has not been systematically tested using multiple species and different HAEs [20].

The specificity of induced defence at the phytohormone, metabolite and herbivore performance levels have been investigated in different plant systems $[5,6,18]$. However, these responses were usually studied separately. It is challenging to integrate the specificity of induced defences at different levels and to understand the underlying mechanisms as they vary amongst different plants [6]. In this study we used a comparative approach to investigate the specificity of the induced responses to three different HAEs among six closely related Nicotiana species at multiple levels, including phytohormones, metabolites and herbivore performance. We specifically focused on three questions: 1) to what extent does one plant species respond to different HAEs? 2) to what extent does one HAE induce different responses among closely related plant species; and 3) does one HAE-induced defence affect insect herbivores differently?

The three HAEs used in this study are: the oral secretions of $M$. sexta $\left(\mathrm{OS}_{M s}\right.$, a specialised feeder on solanaceous plants), the oral secretions of Spodoptera littoralis $\left(\mathrm{OS}_{S b}\right.$, a generalist plant feeder) and $\mathrm{N}$-linolenoyl-glutamic acid (C18:3-Glu, a fatty acid conjugate (FAC) which has been shown to be the most active elicitor in Manduca sexta oral secretions) [21]. The concentration of FACs, including C18:3-Glu, in $\mathrm{OS}_{S l}$ is 500 times lower than that of $\mathrm{OS}_{M s}$ [22]. With these three HAEs we investigated the specificity of induced defence responses in six closely related, diploid $(2 \mathrm{n}=12)$ Nicotiana species that are widely distributed across North and South America: Nicotiana obtusifolia, $N$. linearis, $N$. acuminata, $N$. attenuata, $N$. pauciflora and N. miersii [23] (Figure 1). All species are annuals from the Petunioides clade, except Nicotiana obtusifolia which is a perennial plant from the sister clade Trigonophyllae [24]. Within these six closely related species, $N$. attenuata is an ecological model plant in which the molecular and ecological mechanisms of sophisticated herbivore-induced defence have been studied in detail. Here we use a comparative approach to study the specificity of induced defence from both mechanistic and evolutionary perspectives.

\section{Results}

Induction of JA is both HAE- and species-specific in Nicotiana The pattern of JA accumulation was specific for each HAE studied and the HAE-induced JA accumulation pattern also differed among the Nicotiana species (Figure 2). The analysis revealed that JA accumulation in response to wounding and HAE addition was highly plant speciesand HAE-specific. The highest plant species-specificity of HAE-induced JA induction was found in N. obtusifolia and $N$. pauciflora (Figure 2). In N. obtusifolia, neither C18:3-Glu nor $\mathrm{OS}_{M s}$ affected JA levels, and only $\mathrm{OS}_{S l}$ induced a slight accumulation of JA which was not reflected in higher JA- isoleucine (JA-Ile) levels at $1 \mathrm{~h}$ after elicitation. In contrast to the other five Nicotiana species that showed the highest JA levels at 30 minutes, N. pauciflora 


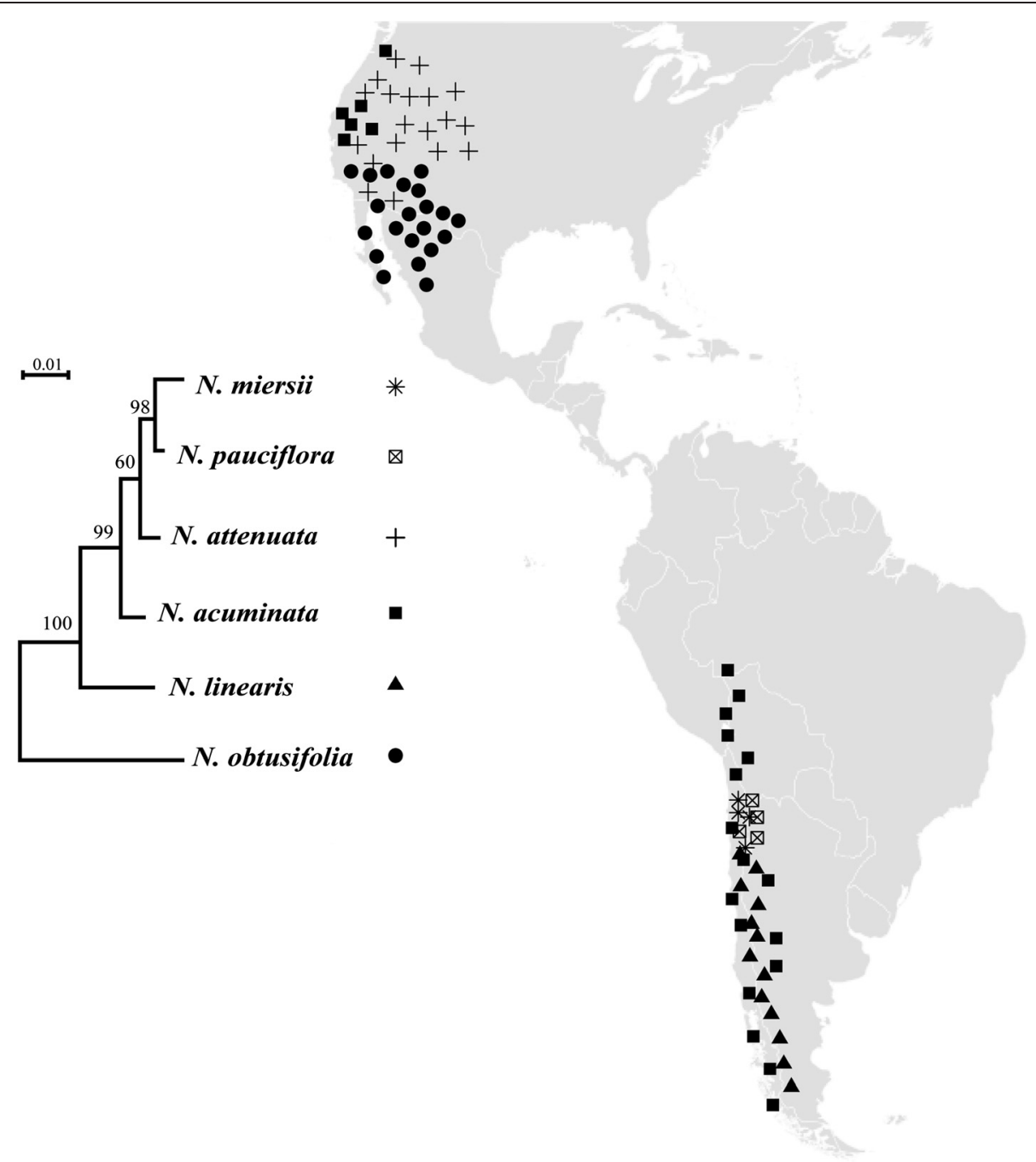

Figure 1 The geographic distribution of six closely related Nicotiana species analysed in this study, redrawn from Goodspeed T. H. [23]. A phylogenetic tree of the six Nicotiana species was constructed from partial nepGS gene sequences obtained from Clarkson et al. 2010 [24], using maximum likelihood method and numbers on each branch represent bootstrap value. Each symbol represents different species. The location of the symbol indicates the distribution of the species that was extracted from Goodspeed T. H [23]. Filled circle: N. obtusifolia; filled triangle: N. linearis; filled square: N. acuminata; plus: N. attenuata; square with cross inside: N. pauciflora; star: N. miersii.

showed peak JA levels at $2 \mathrm{~h}$ after HAE induction (Figure 2).

The specificity HAE was evident when comparing JA responses to the elicitations with $\mathrm{OS}_{M s}$ and $\mathrm{OS}_{S l}$. Elicitations with $\mathrm{OS}_{M s}$ and C18:3-Glu showed similar JA and JA-Ile induction patterns among all six species, which is consistent with a previous study that found C18:3-Glu to be the main component in $\mathrm{OS}_{M s}$ that elicits JA accumulation in Nicotiana. The strongest differences between $\mathrm{OS}_{M s}$ and $\mathrm{OS}_{S l}$ induced JA accumulations were found in N. pauciflora and N. miersii (Figure 2). In $N$. miersii, neither $\mathrm{OS}_{M s}$ nor C18:3-Glu induced any JA accumulations (Figure 2), but $\mathrm{OS}_{S l}$ was highly effective, eliciting JA increases 1.8 times that of a wounding control (wounding + water). This indicates that while C18:3-
Glu elicitation failed to induce JA, N. miersii specifically responds to $\mathrm{OS}_{S l}$. Interestingly, the opposite pattern was found in $N$. pauciflora, in which $\mathrm{OS}_{S l}$ did not induce JA and JA-Ile increases, while both $\mathrm{OS}_{M s}$ and C18:3-Glu induced a more than 20-fold JA increase in comparison to controls (wounding + water). These results suggest that $\mathrm{OS}_{S l}$ may contain FAC-independent elicitors and/or inhibitors. To test this hypothesis, all FACs were removed from $\mathrm{OS}_{S l}$ using an ion exchange column and the FAC-free $\mathrm{OS}_{S l}$ was applied to wounds in N. attenuata leaves, a species showing induced JA accumulation in response to both FAC and $\mathrm{OS}_{S l}$ (Figure 2). The results revealed that FAC-free $\mathrm{OS}_{S l}$ can elicit significant JA accumulations in $N$. attenuata at 30 minutes after treatment confirming the existence of FAC-independent 


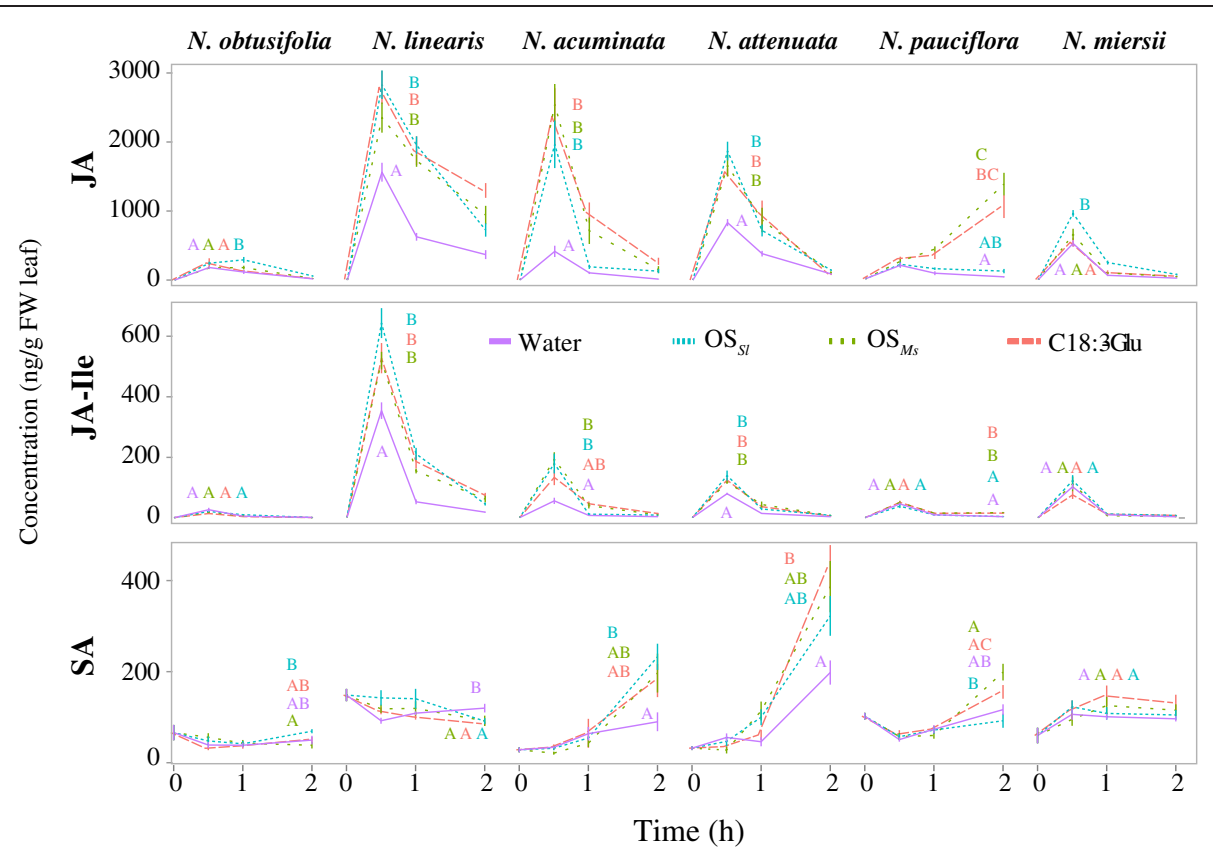

Figure 2 HAE induced phytohormone responses in the different Nicotiana species is both HAE- and species-specific. Each column indicates a different species. The top row represents induced JA responses, the middle row represents induced JA-isoleucine (JA-lle) responses and the bottom row represents induced salicylic acid (SA) responses. Phytohormones were measured at $0 \mathrm{~h}, 0.5 \mathrm{~h}, 1 \mathrm{~h}$ and $2 \mathrm{~h}$ after HAE induction. Different line types represent different treatments. Solid purple colour indicates wounding $(\mathrm{W})+$ water, light blue dotted line indicates $W+S$. littoralis $O S\left(O S_{S l}\right)$ induction; green dashed line indicates $W+M$. sexta OS $\left(\mathrm{OS}_{M S}\right)$ induction, and vermillion dashed line indicates $W+C 18: 3-G l u$ induction. Letters indicate the statistically significant differences $(p<0.05)$ at the peak JA, JA-lle and SA concentrations for each species as determined by ANOVA or Kruskal-Wallis test depending on whether the data were normally distributed or not. The error bar indicates standard error.

elicitors in $\mathrm{OS}_{S l}$ (Figure 3A and B). Furthermore, adding synthetic FAC to FAC-free $\mathrm{OS}_{S l}$ produced a treatment which elicited a similar increase in JA accumulation as that produced by FAC alone, suggesting that $\mathrm{OS}_{S l}$ lacks factors which inhibit FAC responses (Figure 3B). The same result was observed for $N$. pauciflora, in which FAC-complemented $\mathrm{OS}_{S l}$ induced similar JA accumulations to that elicited by FAC and $\mathrm{OS}_{M s}$ (Additional file 1). These results suggest that $\mathrm{OS}_{S l}$ and $\mathrm{OS}_{M s}$ have different elicitor compositions which induce JA accumulations in Nicotiana through different molecular mechanisms. The induced SA levels did not show an HAE-specific pattern (Figure 2).

\section{The induction of trypsin proteinase inhibitor (TPI) activity} differs across different Nicotiana species

The HAE-induced TPI activity varied among the six species investigated (Figure 4A) and correlated with the induction of JA and JA-Ile accumulation (Figure 4B and C). Two species, $N$. miersii and $N$. obtusifolia, which showed only low levels of JA and JA-Ile accumulations within $2 \mathrm{~h}$ after HAE induction, did not show TPI activity changes in comparison to the control at $24 \mathrm{~h}$ after treatment. The other four species, $N$. linearis, $N$. acuminata, $N$. attenuata, and $N$. pauciflora, which showed a high level of JA and JA-Ile accumulation within $2 \mathrm{~h}$ after HAE induction, also showed high induced TPI activity at $24 \mathrm{~h}$ after treatment. All three HAEs induced similar levels of TPI activity and this was consistent among all species with the exception of $N$. pauciflora, where TPI activity was induced by $\mathrm{OS}_{M s}$ and C18:3-Glu but not by $\mathrm{OS}_{S l}$. This also correlates with the observation that only $\mathrm{OS}_{M s}$ and C18:3-Glu induced JA and JA-Ile accumulations in $N$. pauciflora within $2 \mathrm{~h}$ after treatment. Overall HAE-induced TPI activity is highly correlated with the level of induced JA and JA-Ile accumulations and therefore shows a response pattern specific to each Nicotiana species (Figure 4).

\section{HAE-induced resistance to $M$. sexta and $S$. littoralis are HAE-specific, but only induced resistance to $M$. sexta is species-specific}

A detached leaf assay was performed to measure the induced resistance of four Nicotiana species that showed $\mathrm{HAE}$ and species-specific phytohormone responses to the specialist herbivore $M$. sexta and the generalist $S$. littoralis. Induced resistance to $M$. sexta was found to have high species- and HAE-specificity. Among the four tested species only two, $N$. attenuata and $N$. pauciflora, showed induced resistance to $M$. sexta suggesting this induced resistance is species-specific. Interestingly, in both species, only $M$. sexta larvae fed on C18:3-Glu treated leaves gained less mass than those fed on leaves 


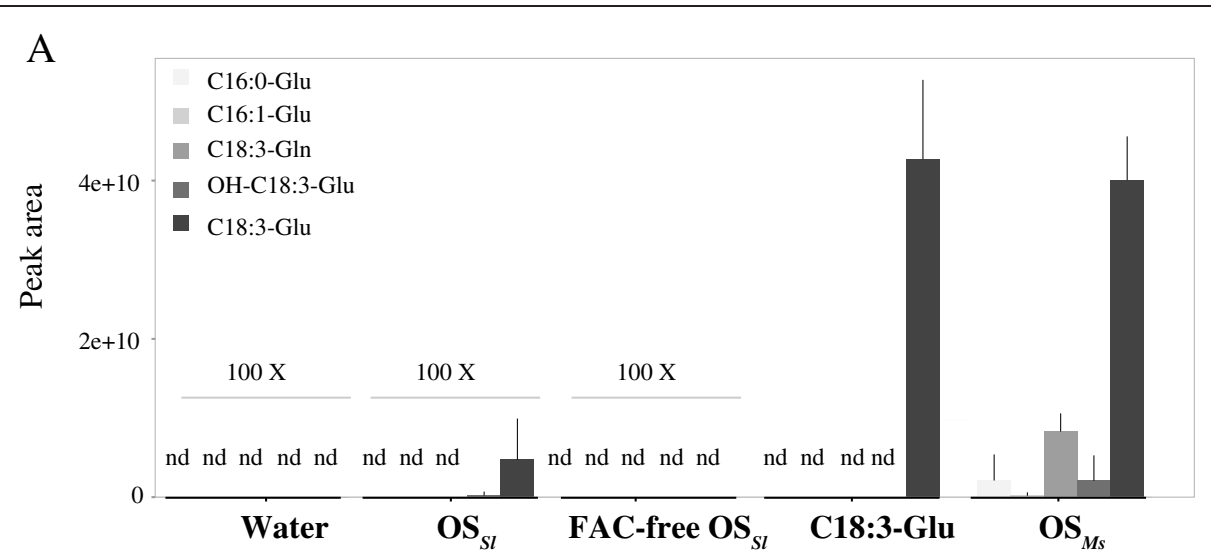

B

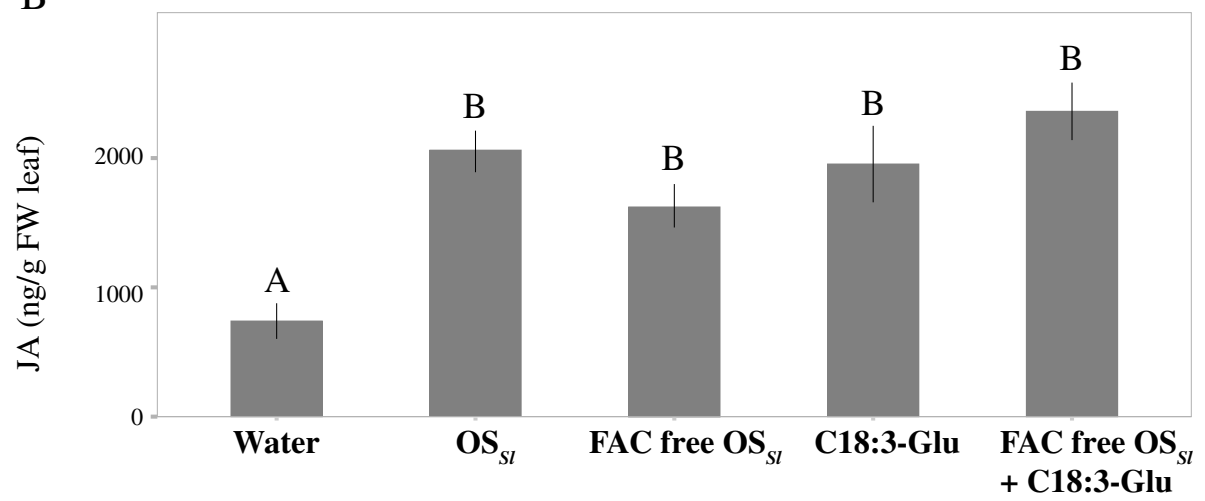

Figure $3 \mathrm{~S}$. littoralis oral secretion (OS) induces JA accumulation independent of the FAC content of the OS. A: The five most abundant FAC compounds were measured by HPLC-MS for all HAEs and control used in this study. Water; oral secretion from S. littoralis $\left(\mathrm{OS}_{S \mathrm{~S})}\right)$; OS $\mathrm{S}_{S /}$ with fatty acid amino acid conjugates (FAC) removed (FAC-free $O_{S S}$ ) using an ion exchange column; C18:3-Glu and oral secretion from $M$. sexta (OS ${ }_{M S}$ ). Each shading represents a specific FAC compound. Y-axis shows the peak area of the target molecular ion of each compound. For each HAE, six replicates were used. Error bar indicates the standard deviation. "nd" refers to samples in which an FAC was not detected. (B) The induced JA accumulation in $\mathrm{N}$. attenuata induced by wounding + oral secretion of S. littoralis. Y-axis shows the JA concentration at 30 minutes after induction and axis refers to different HAEs that were added to leaf punctures. Letters indicates the significance of differences $(p<0.05$, post hoc Tukey honest significant test after ANOVA).

treated with water (control) (Figure 5). The larval mass of $M$. sexta fed on $\mathrm{OS}_{M s}$ or $\mathrm{OS}_{S l}$ treated leaves was not significantly different from the control, indicating the induced resistance is specific to C18:3-Glu.

Different from the induced resistance to $M$. sexta, the induced resistance to $S$. littoralis was found only to be HAE-specific. The induced resistance to $S$. littoralis can be observed in all four species suggesting low species specificity. However, within a species, the level of induced resistance to $S$. littoralis differed amongst the different HAEs (Figure 5), indicating high HAE-specificity within species. In summary, our results showed that the induced resistance to $M$. sexta differs from the induced resistance to $S$. littoralis.

\section{Discussion}

The specificity of HAE-induced defences can be analysed from at least three perspectives: 1) induced defences of one plant species in response to different HAEs; 2) induced defences of different plant species in response to the same HAE; and 3) the effect of the same induced defences on different herbivorous insects. Here we investigated the specificity of HAE-induced defences from all three perspectives.

\section{To which extent does one plant species respond to different HAEs?}

Plants can respond to different HAEs at multiple levels. At the phytohormone level, the six Nicotiana species displayed the same JA responses to C18:3-Glu as to $\mathrm{OS}_{M s}$ but not to $\mathrm{OS}_{S l}$ (Figure 2). This is consistent with a previous study which showed that C18:3-Glu is the elicitor responsible for JA accumulation in $\mathrm{OS}_{M s}$ [21]. Differences in JA accumulation between $\mathrm{OS}_{S l}$ and the other two HAEs are likely due to the different elicitors in $\mathrm{OS}_{S l}$ capable of inducing JA accumulation. N. obtusifolia and $N$. miersii showed no JA responses to C18:3-Glu and $\mathrm{OS}_{M s}$, which is consistent with previous studies $[25,26]$, 


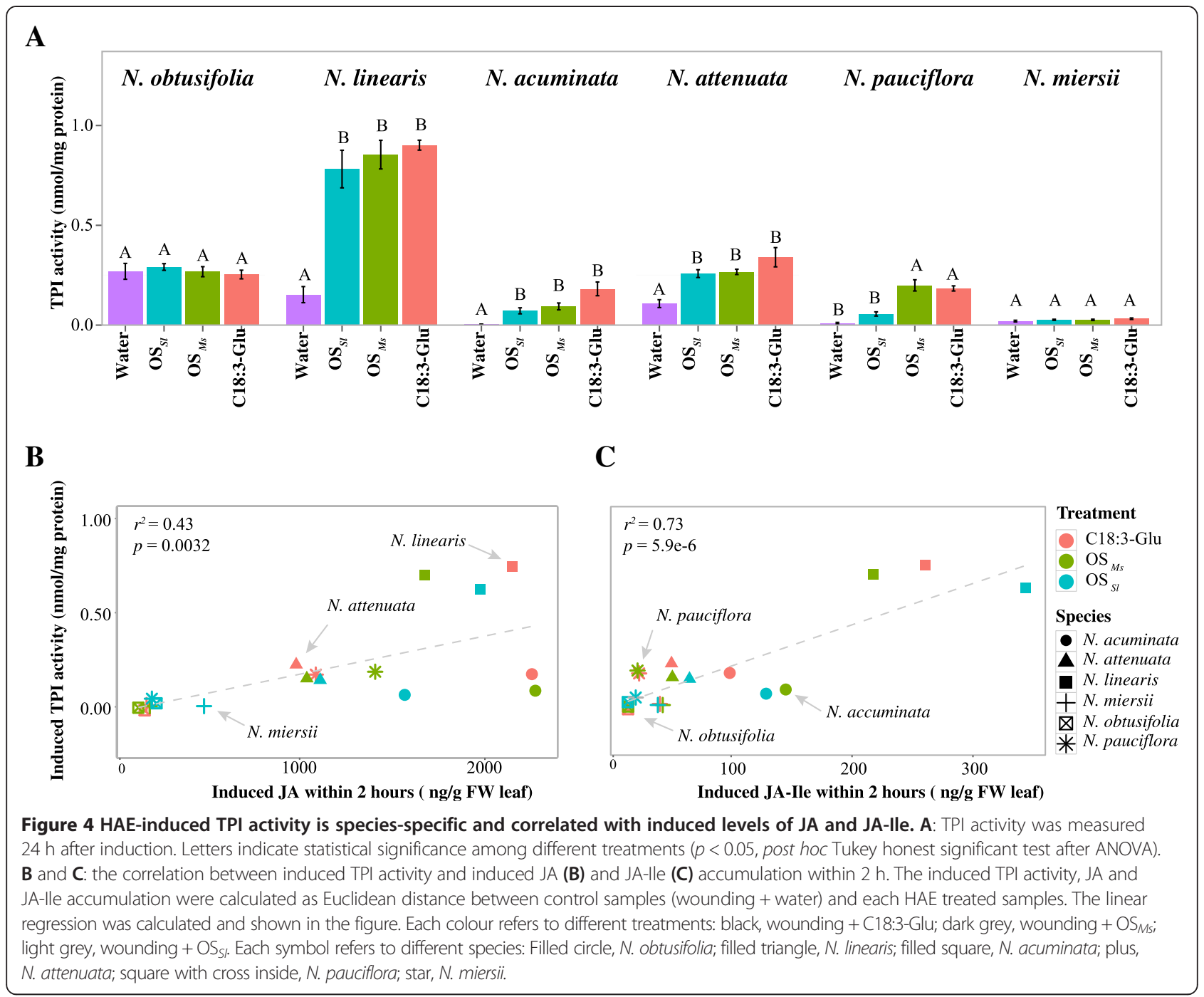

but they did accumulate JA in response to $\mathrm{OS}_{S l}$. In contrast, $N$. pauciflora showed a 20-fold JA increase in response to C18:3-Glu and $\mathrm{OS}_{M s}$ at $2 \mathrm{~h}$ after induction but no JA increase in response to $\mathrm{OS}_{S l}$. These results indicate that the molecular mechanisms of $\mathrm{OS}_{S l}$ perception and subsequent JA responses are likely different from those of $\mathrm{OS}_{M s} / \mathrm{C} 18: 3-\mathrm{Glu}$ perception and responses, since a plant species can lose its perception of one but maintain sensitivity towards others. Indeed our results show that $\mathrm{OS}_{S l}$ induced JA accumulation is independent of FAC, because $\mathrm{OS}_{S l}$ and the FAC-free $\mathrm{OS}_{S l}$ induced the same level of JA accumulation in $N$. attenuata (Figure 3A and B). In addition, $\mathrm{OS}_{S l}$ and FAC-supplemented $\mathrm{OS}_{S l}$ induced similar levels of JA in both $N$. attenuata and $N$. pauciflora. This suggests that the lack of response to $\mathrm{OS}_{S I}$ at the JA level is not due to the presence of an inhibitor in $\mathrm{OS}_{S l}$ (Figure 3 and Additional file 1). Studies have shown that plants can respond to several non-FAC elicitors in the $\mathrm{OS}_{S l}$, such as porin-like proteins (PLP) [27], and oligosaccharides [28]. However, whether these elicitors trigger JA accumulation in Nicotiana species remains unknown. Interestingly, the interaction of JA and SA did not show an HAE-specific pattern (Figure 2). In summary, Nicotiana spp. showed highly-specific induced JA accumulation in response to different HAEs due to their different elicitor compositions and we hypothesize that this may be mediated by differences in receptor-ligand interactions amongst the species.

TPI has been shown to be an important anti-herbivore defence trait. Most Nicotiana species showed similar levels of induced TPI activity in response to the different HAEs at $24 \mathrm{~h}$ after induction (Figure $4 \mathrm{~A}$ ). This suggests that induced TPI activity is not HAE-specific, although further studies on the complete kinetics of HAE-induced TPI activity in all six Nicotiana species are required to confirm this inference. The only exception was found in $N$. pauciflora, which showed increased TPI activity in response to elicitation by $\mathrm{C} 18: 3-\mathrm{Glu}$ and $\mathrm{OS}_{M s}$ but not 


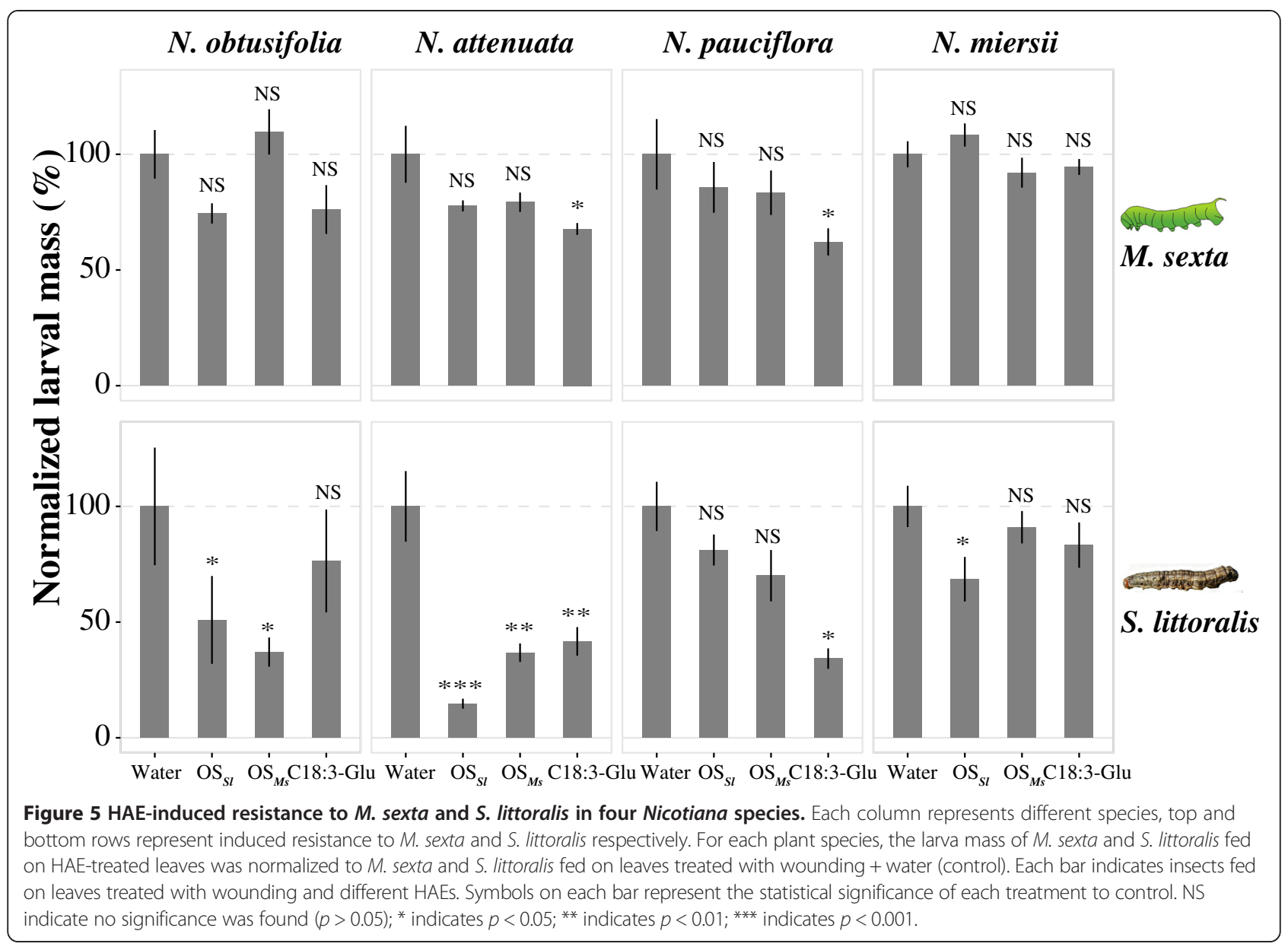

$\mathrm{OS}_{S l}$ (Figure 4A). We conclude that this is largely due to the low level of $\mathrm{OS}_{S l}$-induced JA and JA-Ile accumulation in this species (Figure 2, 5th column), because studies have shown that the induction of TPI activity is regulated by JA-signalling [15,29]. A significant withinspecies correlation between specific HAE induction of JA and the corresponding TPI activity was not found in $N$. miersii and $N$. obtusifolia. These species showed induced JA accumulations only in response to $\mathrm{OS}_{S l}$ (Figure 2, 1st and 6th column) and no induced TPI activity at all (Figure 4A). This is likely because of the low levels of $\mathrm{OS}_{S l}$-induced JA and JA-Ile in N. obtusifolia and N. miersii and such low levels of JA/JA-Ile may not be sufficient to induce TPI activity. Indeed, increasing JA levels by treating $N$. miersii and $N$. obtusifolia with MeJA dramatically increased TPI transcript level and activity $[25,26]$ which is consistent with the hypothesis that the induced TPI activity is dependent on the magnitude and/or duration [30] of JA and JA-Ile induction in Nicotiana.

Induced resistance to $M$. sexta and $S$. littoralis is dependent on different HAEs (Figure 5). In $N$. attenuata and $N$. pauciflora induced resistance to $M$. sexta was found after elicitation with C18:3-Glu but not after elicitation with $\mathrm{OS}_{S l}$ or $\mathrm{OS}_{M s}$. The difference between $\mathrm{OS}_{S l}$ and C18:3-Glu induced resistance to $M$. sexta may be due to different elicitors or suppressors within the $\mathrm{OS}_{S l}$. However, the difference between resistance induced by $\mathrm{OS}_{M s}$ and C18:3-Glu suggests there may be additional components in $\mathrm{OS}_{M s}$ which suppress the induced defence in plants, since the C18:3-Glu used for elicitation was at the same concentration as would be found in $\mathrm{OS}_{M s}$ (Figure 3A). In both $N$. attenuata and $N$. pauciflora, the induced JA and TPI levels were found to be similar between C18:3-Glu and $\mathrm{OS}_{M s}$ treatments (Figures 2 and $4 \mathrm{~A}$, 4th and 5th column) suggesting that putative suppressors in $M$. sexta OS may act downstream of JAsignalling or on other signalling pathways that regulate plant metabolism. Indeed a previous study has revealed that in $N$. attenuata, FAC-free $\mathrm{OS}_{M s}$ and intact $\mathrm{OS}_{M s}$ could induce higher levels of several protein peptides, such as fragments of RuBPCase activase (RCA) compared to FAC alone [31]. In addition RCA recently has been shown to play an important role not only in photosynthesis but also in JA-mediated growth-defence trade-offs [32]. Therefore $M$. sexta may employ some unknown 
components in its oral secretion to manipulate the growth-defence equilibrium in its host for its own benefit. Cases of insect-suppression of plant-induced defence through components of oral secretion have been reported in a few studies. For example, the OS of S. littoralis and Pieris brassicae were found to suppress wound-induced defence responses in Arabidopsis thaliana [33] and Leptinotarsa decemlineata exploits orally-secreted bacteria to suppress defence in tomato [34]. However, studies have also shown that suppression of direct defences by insect oral secretion can also be beneficial for the plant by reducing the cost of direct defences and increasing plant fitness $[35,36]$. This indicates that in some cases the suppression of induced direct defence by insect oral secretion might be adaptive for both plant and herbivore.

Induced resistance to $S$. littoralis was also shown to be HAE-specific within species (Figure 5). In all four Nicotiana species tested, the induced resistance to $S$. littoralis attack was different for each HAE. Furthermore the variation of induced resistance to $S$. littoralis did not appear to correlate with induced JA accumulation. For example in $N$. attenuata, while both $\mathrm{OS}_{M s}$ and $\mathrm{OS}_{S l}$ treatments showed similar levels of induced JA, the resistance to $S$. littoralis is higher in $\mathrm{OS}_{S l}$ induced samples than $\mathrm{OS}_{M s}$. The same is true for $N$. pauciflora when treated with C18:3-Glu and $\mathrm{OS}_{M s}$, the treatments showed a similar induction of JA accumulation but different induced resistance to S. littoralis. These results suggest that the specificity of induced resistance to $S$. littoralis may also be regulated by other signalling pathways or their cross-talk.

In summary Nicotiana spp. show highly specific induceddefence responses to different HAEs and this is may be due to differences in their elicitor compositions.

To which extent does one HAE induce different responses among closely related species?

The same HAE elicited different responses among the six Nicotiana species. At the phytohormone level, both C18:3-Glu and $\mathrm{OS}_{M s}$ induced JA accumulation in four species. JA accumulation was not induced in $N$. miersii and $N$. obtusifolia which is consistent with previous studies $[25,26]$. Interestingly in both species, $\mathrm{OS}_{S l}$ induced a certain level of JA accumulation (Figure 2). This suggests that these species have intact JA signalling and biosynthesis pathways which can be only specifically activated by some unknown elicitors in $\mathrm{OS}_{S l}$. It is therefore reasonable to assume that $N$. obtusifolia and $N$. miersii have lost the ability to perceive C18:3-Glu.

Changes in $\mathrm{OS}_{S l}$ perception were also found among Nicotiana species. While $\mathrm{OS}_{S l}$ induced JA accumulation in the other five species it did not induce JA accumulation in N. pauciflora (Figure 2, top row, 5th column). As discussed above, it is likely that $N$. pauciflora has lost its perception of non-FAC, $\mathrm{OS}_{S l}$ specific elicitors. Although
HAE-induced JA accumulation and JA-mediated defences have been investigated intensively [2,37], the mechanisms of HAE perception remain unknown. The closely related Nicotiana species possessing intact JA induction and signalling pathways, but lacking the ability to perceive specific HAEs, offer an ideal system for investigating the molecular mechanisms of HAE perception.

The HAE-induced TPI activity varied among Nicotiana species and the variation is correlated with the induced level of JA and JA-Ile (Figure 4B and C). $N$. attenuata, $N$. acuminata, $N$. linearis and $N$. pauciflora, showed high levels of HAE-induced JA accumulation and correspondingly high levels of induced TPI activity (Figure 4A). However $N$. obtusifolia and N. miersii, which had little or no induced JA accumulation, showed no induced TPI activity (Figure 4A). This indicates that, at the species level, induced JA and JA-Ile play central roles in the differences in induced TPI activity among different species.

While induced resistance to $S$. littoralis was found in all four Nicotiana species tested, only two species showed induced resistance to $M$. sexta. The variation of HAEinduced resistance to $M$. sexta among closely related Nicotiana species could be due to differences in their natural histories. For N. obtusifolia, although it has low levels of induced defence, it has high levels of constitutive defence, such as TPI and HGL-DTG, which severely affect M. sexta performance [38]. Therefore, $N$. obtusifolia may have employed a high constitutive defence strategy rather than an induced defence strategy to defend against herbivores. Perhaps this would be expected of a perennial plant that grows in specialized niches along canyon walls and probably rarely faces the type of strong intra-specific competition which is thought to have selected for the elaborate induced defence system of $N$. attenuata [35].

Interestingly, $N$. miersii has both low constitutive and induced defence and is the fastest growing plant among the six species studied (glasshouse observation). This is consistent with $N$. miersii's low level of TPI activity (Figure 4A) [25], as high TPI activity has been shown to slow plant development and reduce fitness in $N$. attenuata [39]. In addition the level of constitutive and induced defence compounds, such as nicotine, nornicotine, caffeoylputrescine and HGL-DTG were very low in N. miersii [25]. This is consistent with the hypothesis that this species may have evolved a high growth - low defence strategy. Indeed under low herbivore pressure, selection on plants will favour a high competitive ability, such as that provided by high growth rate [40,41]. Although little is known about the herbivore composition of its natural habitat, we predict that $N$. miersii has lost its induced defence against $M$. sexta due to low herbivore pressure, or an exceptionally high level of intra-specific competition. Thus, the variation of induced defences among Nicotiana species is thought to result from 
differences in their natural histories and selection pressures. Taken together, our data show that one HAE can induce different defence responses among closely related species at all different levels. This indicates that induced defences can evolve rapidly in Nicotiana. Indeed, even within the species of $N$. attenuata, two genotypes showed different $\mathrm{OS}_{M s}$ induced early defence signalling [42]. The analysis of large numbers of $N$. attenuata isolates collected from different natural populations also suggested that $\mathrm{OS}_{M s}$ induced JA and JA-Ile levels were indeed variable but largely followed a Gaussian distribution within $N$. attenuata (Additional file 2 and Li. et al. Submitted). However, the inter-species variations that were found in this study are much greater than intra-species variation, at least at the level of $\mathrm{OS}_{M s}$ induced JA and JA-Ile levels (Additional file 2).

\section{Does one induced defence affect insect herbivores differently?}

In Nicotiana spp., while C18:3-Glu induced defences negatively affected the growth of both $M$. sexta and $S$. littoralis; the other two HAEs could only reduce the growth rate of $S$. littoralis (Figures 5 and 6). This may be due to differences in the susceptibility of the specialist $M$. sexta and generalist $S$. littoralis to induced plant defences. Because of the long co-evolutionary history between specialists and their hosts, specialists are more able to tolerate and detoxify their host's defence compounds than generalists [20]. Therefore it is likely that while many different induced defence responses can affect the growth rate of $S$. littoralis, the growth rate of $M$. sexta can only be affected by specific, or combinations of specific, defence traits. This hypothesis is supported by the correlation between the induction of TPI (a specialised Nicotiana defence trait) and the increased efficacy of defence against $M$. sexta and S. littoralis. The multiple-domain TPI gene has been shown to be specific to the Nicotiana genus and it is reasonable to posit that it evolved as a consequence of a plant-insect arms race $[43,44]$. The induced resistance to $M$. sexta shown by C18:3-Glu treated $N$. attenuata and $N$. pauciflora was correlated with induced TPI activity (Figures 4A and 5) indicating that induction of TPI might be necessary (but not sufficient) to reduce the growth of $M$. sexta. However species such as $N$. obtusifolia showed induced resistance to $S$. littoralis but did not show TPI activity after elicitation with $\mathrm{OS}_{M s}$ and $\mathrm{OS}_{S l}$ (Figures 5 and 6). This suggests that TPI induction is not required to reduce the growth rate of $S$. littoralis. In summary our data suggests that same induced defence response affects specialist and generalist insect herbivores differently.

\section{Conclusions}

Our systematic investigation of HAE-induced defence in Nicotiana showed that in these species induced defence is highly specific at all three analysed perspectives: a single Nicotiana species can show different defence responses to different HAEs; one HAE can induce different responses among closely related species and the effect of one HAEinduced defence response differs among herbivorous insects. Furthermore, the analysis also indicates that HAE-induced defence can evolve rapidly, because the same HAE can induce divergent responses among closely related Nicotiana species. These closely related species showing distinct HAE-induced defences are therefore an ideal system for the future study of the molecular mechanisms and evolutionary divergence of herbivore-induced defence.

\section{Methods}

\section{Plant growth and sample treatments}

The seeds of different Nicotiana species were either originally collected from natural populations or obtained from the US Nicotiana Germplasm Collection, North Carolina. Nicotiana attenuata Torr. Ex S. Watson and $N$. obtusifolia seeds were originally collected in Utah (USA) and inbred in the glasshouse for 30 generations and 1 generation respectively. The other four Nicotiana species were originally obtained from the US Nicotiana Germplasm Collection and inbred for one generation in the glasshouse. The detailed accession numbers and availability of seeds for all plant species used in our experiments are provided in the Additional file 3. All seeds were germinated according to the $N$. attenuata germination protocol [45]. Ten-day-old seedlings were planted into soil in Teku pots (Waalwijk) and, once established, transferred to $1 \mathrm{~L}$ pots in soil and grown in a York Chamber under a $16 / 8 \mathrm{~h}$ light/dark, $26^{\circ} \mathrm{C}$, and $65 \%$ relative humidity regime until they were in the rosette stage.

$M$. sexta and S. littoralis oral secretions (OS) were collected on ice from larvae reared on $N$. attenuata plants until third to fifth instar as previously described [21]. To analyse the FAC components in the OS each sample was diluted 1:100 $\left(\mathrm{OS}_{M s}\right)$ or 1:10 $\left(\mathrm{OS}_{S l}\right)$ with $15 \%$ methanol and analysed directly on a high-performance liquid chromatograph-mass spectrometer (HPLC-MS) (1200 L LC-MS, Varian, Palo Alto, CA, USA) [21]. FAC-free $\mathrm{OS}_{S l}$ was obtained using the method described in [21]. The synthetic C18:3-Glu used in this study was diluted to $138 \mathrm{ng} \mu \mathrm{L}^{-1}$, which is the same concentration as that found in $\mathrm{OS}_{M s}$.

To simulate herbivore attack one leaf of each plant was wounded with a pattern wheel and $20 \mu \mathrm{L}$ of 1:5 diluted $\mathrm{OS}_{M s}$ or $\mathrm{OS}_{S l}$ or C18:3-Glu or water was added to the puncture wounds. Leaf samples were collected at $0 \mathrm{~h}, 0.5 \mathrm{~h}, 1 \mathrm{~h}$ and $2 \mathrm{~h}$ for phytohormone analysis. Samples for trypsin proteinase inhibitor (TPI) assays were collected $24 \mathrm{~h}$ after treatment. The middle vein of the leaf was excised. All samples were collected in $2 \mathrm{~mL}$ Eppendorf 
A

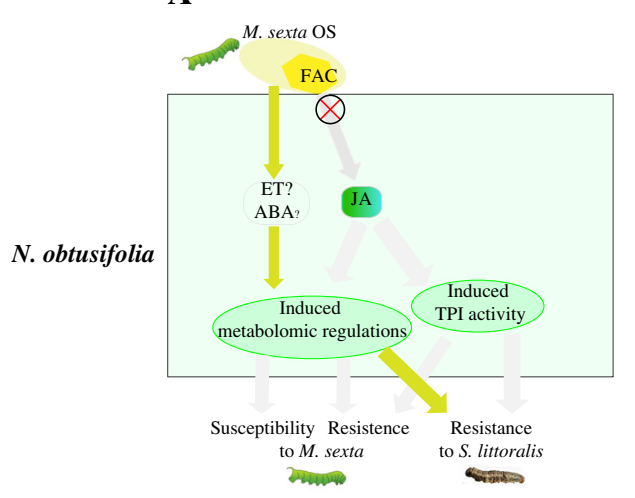

B

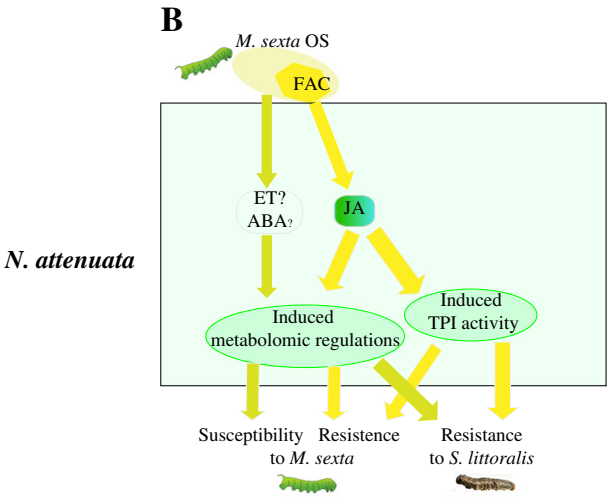

C

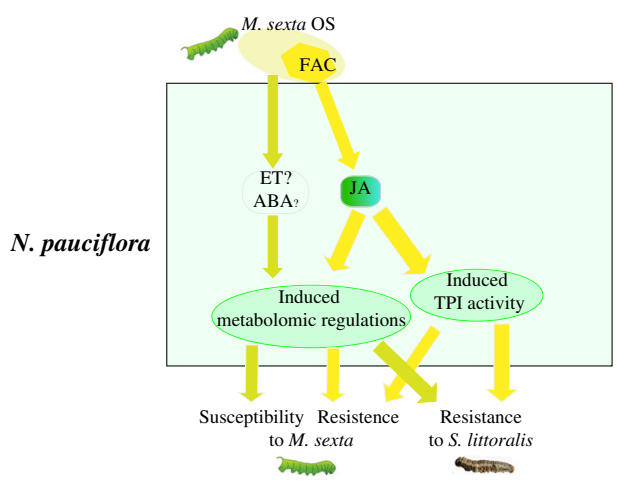

D

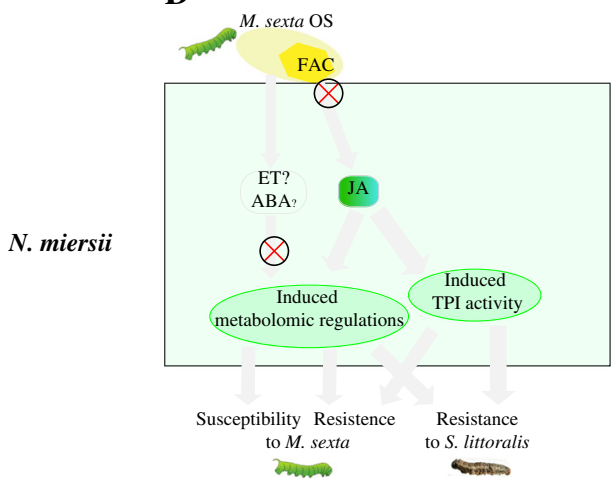

E

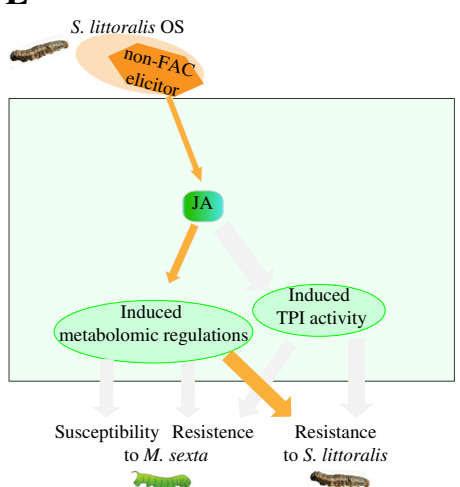

F

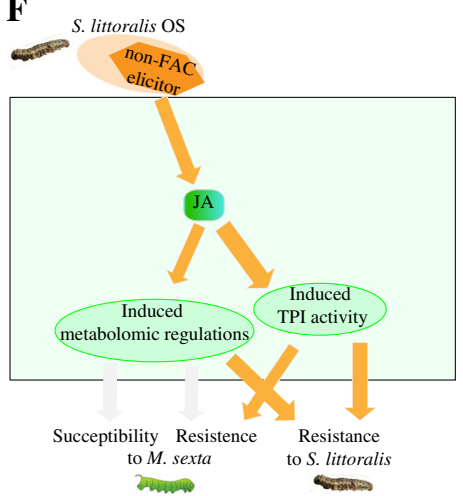

G

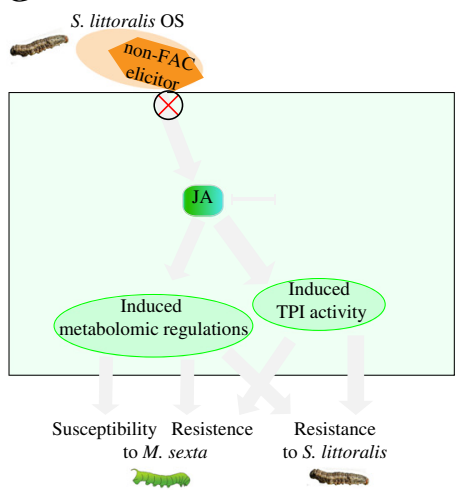

H

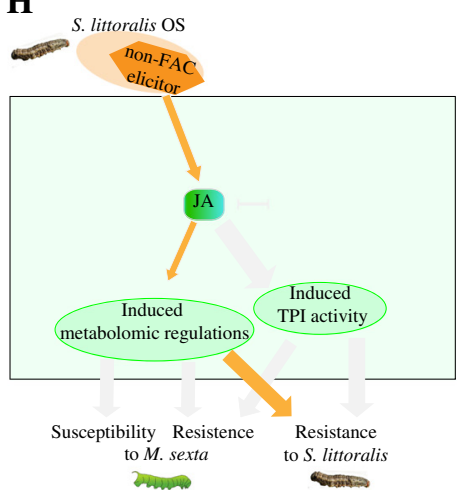

Figure 6 (See legend on next page.) 
(See figure on previous page.)

Figure 6 A model summarizing the evidence for specificity of HAE-induced defences in four Nicotiana species. The coloured arrows indicate the interaction between two components. The colours refers to different HAEs and the filling amount of arrow indicates the strength of an interaction. Grey arrow indicates that no interaction was found. Each panel represents the induced defence model in different species. Red cross in the circle indicates a putative loss of function mutation. $\mathbf{A}-\mathbf{D}$ and $\mathbf{E}-\mathbf{H}$ refer to the $\mathbf{M}$. sexta OS (A-D) and S. littoralis (E-H) induced defence responses in N. obtusifolia (A and E), N. attenuata (B and F), N. pauciflora (C and G) and N. miersii (D and H) respectively. JA: jasmonic acid; ET: ethylene; ABA: abscisic acid; OS: oral secretion; TPI: trypsin proteinase inhibitor.

tubes, flash frozen in liquid nitrogen then stored at $-80^{\circ} \mathrm{C}$ until analysed.

\section{Phytohormone and TPI activity measurements}

Phytohormones were quantified as previously described [15]. In brief, the ground tissues were extracted with ethyl acetate spiked with $100 \mathrm{ng} / \mathrm{mL}$ of 9,10-dideutero-9,10dihydro jasmonic acid (JA-D2), $20 \mathrm{ng} / \mathrm{mL}$ jasmonoyl isoleucine (JA-Ile-13C6), $20 \mathrm{ng} / \mathrm{mL}$ hexadeutero abscisic acid (ABA-D6) and $20 \mathrm{ng} / \mathrm{mL}$ 3,4,5,6-tetradeutero salicylic acid (SA-D4) as internal standards (ISs). Then the extracts were re-suspended in 70\% methanol and the phytohormone content was analysed via HPLC-MS (1200 L LCMS, Varian, Palo Alto, CA, USA) [15]. TPI activity was analysed in $100 \mathrm{mg}$ of leaf tissue with a radial diffusion assay as described by Van Dam et al [46].

\section{Induced resistance detached leaf assay}

To prevent insect feeding effects that may mask HAEinduced responses, the HAE-induced resistance to both $M$. sexta and $S$. littoralis was measured through bioassays using detached leaf feeding. M. sexta were obtained from in-house colonies and $S$. littoralis were obtained from Syngenta (Stein, Switzerland). All bioassays were carried out in an insect chamber (Snijders Scientific, Tilburg, The Netherlands) at $16 / 8 \mathrm{~h}$ light/dark, $26^{\circ} \mathrm{C}$, $80 \%$ humidity and $100 \mu \mathrm{mol} \mathrm{m} \mathrm{m}^{-2} \mathrm{~s}^{-1}$ light intensity. The bioassays for the four treatments in each species were performed at the same time of day in the same insect chamber. For each plant species, rosette stage leaves (leaves at node +1 ) were wounded and treated with $\mathrm{OS}_{M s}, \mathrm{OS}_{S l}, \mathrm{C} 18: 3-\mathrm{Glu}$ or water (control) at $11 \mathrm{am}$. After $24 \mathrm{~h}$, the treated leaves were cut and mounted in $2 \mathrm{~mL}$ Eppendorf tubes filled with moist cotton, then placed in a vertical position inside clear polystyrene food boxes. The leaves were replaced every two days with new leaves from plants that were treated with the same HAE $24 \mathrm{~h}$ previously. To ensure all insect larvae were at a similar developmental stage and with the same body mass at the start of each bioassay, the newly hatched neonate $(M$. sexta) or first instar larvae (S. littoralis, fed on artificial diet) were first placed on the untreated leaves of the test species for $48 \mathrm{~h}$. Then the neonates were weighed and 20 neonates with similar mass (6-10 $\mathrm{mg}$ and $1.8-3.3 \mathrm{mg}$ for $M$. sexta and S. littoralis respectively) were selected and used for the bioassays. For $M$. sexta the bioassays were stopped after 5 days. For $S$. littoralis, the bioassays lasted 6 days, except the assays on $N$. pauciflora and $N$. miersii which lasted only 5 days because the leaves of these species are not large enough to feed S. littorals beyond this time. At the end of the bioassay all surviving larvae were weighed. To make the induced resistance comparable among different species the body mass was normalized to the average larval body mass of the control group (fed on Wounding + Water treated leaves) for each bioassay.

\section{Statistical analysis}

All statistical analyses and non-targeted metabolomic analyses were performed in $\mathrm{R}$ version 3.0.1 [47]. The significance of phytohormone and TPI activity induced by different HAEs was assessed by parametric or nonparametric tests after normality testing of the data distribution by the Shapiro test. For parametric tests ANOVA and post hoc Tukey honest significant difference (HSD) tests were carried out by using the "aov" and "TukeyHSD” functions. For non-parametric tests, KruskalWallis and associated multiple-comparison tests were carried out by using the "kruskalmc" function in the "pgirmess" library. The significance of induced resistance between HAE and control was assessed by the Student's- $t$ test or the Mann-Whitney U test after normality testing of data distribution by the Shapiro test.

\section{Availability of supporting data}

The data sets supporting the results of this article are available in the Labarchives repository: http://doi.org/ 10.6070/H4DF6P6W.

\section{Additional files}

Additional file 1: C18:3-Glu supplemented $S$. littoralis OS $\left(\operatorname{OS}_{S_{I}}\right)$ induced JA accumulations in N. pauciflora to levels equivalent to those induced by $M$. sexta OS (OS $\left.\mathrm{OS}_{M s}\right)$. JA was measured at two hours after the wounding plus OS treatment when $N$. pauciflora showed the highest induced JA accumulation. SS $_{S /}$ was supplemented with an amount of C18:3-Glu equivalent to that found in M. sexta OS (OS $\mathrm{S}_{\text {S }}$ ). NS indicates no statistical difference was found in comparison to control (wounding + water). Letter indicates statistical significance was found in comparison to control ( $p<0.05$, post hoc Tukey honest significant test after ANOVA).

Additional file 2: The variation of $O S_{M s}$ induced JA accumulations among closely related Nicotiana species is much greater than different $\boldsymbol{N}$. attenuata accessions. The level of JA and JA-lle accumulation was measured at one hour after ${ }_{0} S_{M s}$ treatment. In order to compare the 
intra-species and inter-species variation, we normalized the JA level to $\mathrm{N}$ attenuata UT accession (30th in bred), which was used in both datasets. Red color refers to closely related Nicotiana species that were used in this study, blue color refers to different accessions of $\mathrm{N}$. attenuata collected from natural population. The detailed sample information of different $N$. attenuata is included in another publication (Li. et. al. submitted). A: the density plot of OS ${ }_{M S}$ induced JA level; B: the distribution of $\mathrm{OS}_{M S}$ induced JA-lle level.

Additional file 3: The stock centre numbers and availability of plants that were used in this study.

\section{Abbreviations}

HAE: Herbivore associated elicitor; OS: Oral secretion; FAC: Fatty acid amino acid conjugate; JA: Jasmonic acid; TPI: Trypsin protease inhibitor.

\section{Competing interests}

The authors declare that they have no competing interests.

\section{Authors' contributions}

SX concieved and performed experiments, analysed the data and wrote the manuscript; WZ performed experiments and analysed data; SP performed experiments; ITB contributed reagents, materials, analysis tools and provided suggestions on the experimental design. All authors contributed in revising the manuscript and approved the final manuscript.

\section{Acknowledgments}

We thank T. Krügel for providing the seeds of different Nicotiana species, S. Meldau for constructive discussions at the beginning of this project, M. Kallenbach for providing synthetic C18:3-Glu, and M. Schäfer for the help with the TPI activity assay. We also thank A. Navarro, M. Huber, M. Schuman and M. Durrant for their critical comments on the manuscript, and H. Guo for the constructive suggestions on the experiments and the manuscript. We thank three anonymous reviewers for providing constructive suggestions on the manuscript. We are grateful for the funding by Swiss National Science Foundation (Project number PEBZP3-142886 to SX), the Marie Curie IntraEuropean Fellowships (IEF) (Project Number 328935 to SX), the Max Planck Society and European Research Council advanced grant ClockworkGreen (Project number 293926 to ITB).

\section{Received: 6 July 2014 Accepted: 22 December 2014}

\section{Published online: 16 January 2015}

\section{References}

1. Karban R, Baldwin IT. Induced responses to herbivory. Chicago: University of Chicago Press; 1997.

2. Bonaventure G, VanDoorn A, Baldwin IT. Herbivore-associated elicitors: FAC signaling and metabolism. Trends Plant Sci. 2011;16(6):294-9.

3. Bingham RA, Agrawal AA. Specificity and trade-offs in the induced plant defence of common milkweed Asclepias syriaca to two lepidopteran herbivores. J Ecol. 2010;98(5):1014-22.

4. Erb M, Meldau S, Howe GA. Role of phytohormones in insect-specific plant reactions. Trends Plant Sci. 2012;17(5):250-9.

5. Van Zandt PA, Agrawal AA. Specificity of induced plant responses to specialist herbivores of the common milkweed Asclepias syriaca. Oikos. 2004;104(2):401-9.

6. Schmelz EA, Engelberth J, Alborn HT, Tumlinson JH, Teal PE. Phytohormonebased activity mapping of insect herbivore-produced elicitors. Proc Natl Acad Sci U S A. 2009;106(2):653-7.

7. Farmer EE, Ryan CA. Interplant communication - airborne methyl jasmonate induces synthesis of proteinase-inhibitors in plant-leaves. Proc Natl Acad Sci U S A. 1990;87(19):7713-6.

8. Ballare CL. Jasmonate-induced defenses: a tale of intelligence, collaborators and rascals. Trends Plant Sci. 2011;16(5):249-57.

9. Pieterse CM, Leon-Reyes A, Van der Ent S, Van Wees SCM. Networking by small-molecule hormones in plant immunity. Nat Chem Biol. 2009;5(5):308-16.

10. Thaler JS, Humphrey PT, Whiteman NK. Evolution of jasmonate and salicylate signal crosstalk. Trends Plant Sci. 2012;17(5):260-70.

11. Diezel C, von Dahl CC, Gaquerel E, Baldwin IT. Different lepidopteran elicitors account for cross-talk in herbivory-induced phytohormone signaling. Plant Physiol. 2009;150(3):1576-86.

12. Truitt $\mathrm{CL}$, Wei HX, Pare PW. A plasma membrane protein from Zea mays binds with the herbivore elicitor volicitin. Plant Cell. 2004;16(2):523-32.
13. Heiling S, Schuman MC, Schoettner M, Mukerjee P, Berger B, Schneider B, et al. Jasmonate and ppHsystemin regulate key malonylation steps in the biosynthesis of 17-hydroxygeranyllinalool diterpene glycosides, an abundant and effective direct defense against herbivores in Nicotiana attenuata. Plant Cell. 2010;22(1):273-92.

14. Zavala JA, Patankar AG, Gase K, Hui D, Baldwin IT. Manipulation of endogenous trypsin proteinase inhibitor production in Nicotiana attenuata demonstrates their function as antiherbivore defenses. Plant Physiol. 2004;134(3):1181-90.

15. Wang L, Halitschke R, Kang JH, Berg A, Harnisch F, Baldwin IT. Independently silencing two JAR family members impairs levels of trypsin proteinase inhibitors but not nicotine. Planta. 2007:226(1):159-67.

16. Keinanen M, Oldham NJ, Baldwin IT. Rapid HPLC screening of jasmonateinduced increases in tobacco alkaloids, phenolics, and diterpene glycosides in Nicotiana attenuata. J Agric Food Chem. 2001;49(8):3553-8.

17. Winz RA, Baldwin IT. Molecular interactions between the specialist herbivore Manduca sexta (Lepidoptera, Sphingidae) and its natural host Nicotiana attenuata. IV. Insect-induced ethylene reduces jasmonate-induced nicotine accumulation by regulating putrescine $\mathrm{N}$-methyltransferase transcripts. Plant Physiol. 2001;125(4):2189-202.

18. Agrawal AA. Specificity of induced resistance in wild radish: causes and consequences for two specialist and two generalist caterpillars. Oikos. 2000;89(3):493-500.

19. Agrawal AA. Induced responses to herbivory in wild radish: effects on several herbivores and plant fitness. Ecology. 1999;80(5):1713-23.

20. Ali JG, Agrawal AA. Specialist versus generalist insect herbivores and plant defense. Trends Plant Sci. 2012:17(5):293-302.

21. Halitschke R, Schittko U, Pohnert G, Boland W, Baldwin IT. Molecular interactions between the specialist herbivore Manduca sexta (Lepidoptera, Sphingidae) and its natural host Nicotiana attenuata. III. Fatty acid-amino acid conjugates in herbivore oral secretions are necessary and sufficient for herbivore-specific plant responses. Plant Physiol. 2001;125(2):711-7.

22. Hettenhausen C, Baldwin IT, Wu JQ. Nicotiana attenuata MPK4 suppresses a novel jasmonic acid (JA) signaling-independent defense pathway against the specialist insect Manduca sexta, but is not required for the resistance to the generalist Spodoptera littoralis. New Phytol. 2013;199(3):787-99.

23. Goodspeed TH. The genus Nicotiana. Chronica Botanica. 1954;16(536):102-35.

24. Clarkson JJ, Kelly $\sqcup$, Leitch AR, Knapp S, Chase MW. Nuclear glutamine synthetase evolution in Nicotiana: phylogenetics and the origins of allotetraploid and homoploid (diploid) hybrids. Mol Phylogenet Evol. 2010;55(1):99-112.

25. Pearse IS, Krugel T, Baldwin IT. Innovation in anti-herbivore defense systems during neopolypoloidy - the functional consequences of instantaneous speciation. Plant J. 2006:47(2):196-210.

26. Anssour S, Baldwin IT. Variation in antiherbivore defense responses in synthetic Nicotiana allopolyploids correlates with changes in uniparental patterns of gene expression. Plant Physiol. 2010;153(4):1907-18.

27. Guo HJ, Wielsch N, Hafke JB, Svatos A, Mithofer A, Boland W. A porin-like protein from oral secretions of Spodoptera littoralis larvae induces defenserelated early events in plant leaves. Insect Biochem Mol. 2013;43(9):849-58.

28. Mack L, Gros P, Burkhardt J, Seifert K. Elicitors of tansy volatiles from cotton leafworm larval oral secretion. Phytochemistry. 2013:96:158-69.

29. Paschold A, Halitschke R, Baldwin IT. Co(i)-ordinating defenses: NaCOI 1 mediates herbivore- induced resistance in Nicotiana attenuata and reveals the role of herbivore movement in avoiding defenses. Plant J. 2007;51(1):79-91.

30. Stork W, Diezel C, Halitschke R, Galis I, Baldwin IT. An ecological analysis of the herbivory-elicited JA burst and its metabolism: plant memory processes and predictions of the moving target model. PLoS One. 2009:4(3):e4697.

31. Giri AP, Wunsche H, Mitra S, Zavala JA, Muck A, Svatos A, et al. Molecular interactions between the specialist herbivore Manduca sexta (Lepidoptera, Sphingidae) and its natural host Nicotiana attenuata. VII. Changes in the plant's proteome. Plant Physiol. 2006;142(4):1621-41.

32. Mitra S, Baldwin IT. RuBPCase activase (RCA) mediates growth-defense trade-offs: silencing RCA redirects jasmonic acid (JA) flux from JA-isoleucine to methyl jasmonate (MeJA) to attenuate induced defense responses in Nicotiana attenuata. New Phytol. 2014;201(4):1385-95.

33. Consales F, Schweizer F, Erb M, Gouhier-Darimont C, Bodenhausen N, Bruessow $F$, et al. Insect oral secretions suppress wound-induced responses in Arabidopsis. J Exp Bot. 2012;63(2):727-37.

34. Chung SH, Rosa C, Scully ED, Peiffer M, Tooker JF, Hoover K, et al. Herbivore exploits orally secreted bacteria to suppress plant defenses. Proc Natl Acad Sci U S A. 2013;110(39):15728-33. 
35. Voelckel C, Schittko U, Baldwin IT. Herbivore-induced ethylene burst reduces fitness costs of jasmonate- and oral secretion-induced defenses in Nicotiana attenuata. Oecologia. 2001;127(2):274-80.

36. Kahl J, Siemens DH, Aerts RJ, Gabler R, Kuhnemann F, Preston CA, et al. Herbivore-induced ethylene suppresses a direct defense but not a putative indirect defense against an adapted herbivore. Planta. 2000;210(2):336-42.

37. Wu J, Baldwin IT. New insights into plant responses to the attack from insect herbivores. Annu Rev Genet. 2010;44:1-24.

38. Jassbi AR, Zamanizadehnajari S, Baldwin IT. 17-Hydroxygeranyllinalool glycosides are major resistance traits of Nicotiana obtusifolia against attack from tobacco hornworm larvae. Phytochemistry. 2010;71(10):1115-21.

39. Zavala JA, Patankar AG, Gase K, Baldwin IT. Constitutive and inducible trypsin proteinase inhibitor production incurs large fitness costs in Nicotiana attenuata. Proc Natl Acad Sci U S A. 2004;101(6):1607-12.

40. Agrawal AA, Hastings AP, Johnson MTJ, Maron JL, Salminen JP. Insect herbivores drive real-time ecological and evolutionary change in plant populations. Science. 2012;338(6103):113-6.

41. Zust T, Heichinger C, Grossniklaus U, Harrington R, Kliebenstein DJ, Turnbull LA. Natural enemies drive geographic variation in plant defenses. Science. 2012;338(6103):116-9.

42. Wu JQ, Hettenhausen C, Schuman MC, Baldwin IT. A comparison of two Nicotiana attenuata accessions reveals large differences in signaling induced by oral secretions of the specialist herbivore Manduca sexta. Plant Physiol. 2008;146(3):927-39.

43. Wu J, Hettenhausen C, Baldwin IT. Evolution of proteinase inhibitor defenses in North American allopolyploid species of Nicotiana. Planta. 2006;224 (4):750-60.

44. Barta E, Pintar A, Pongor S. Repeats with variations: accelerated evolution of the Pin2 family of proteinase inhibitors. Trends Genet. 2002;18(12):600-3.

45. Krugel T, Lim M, Gase K, Halitschke R, Baldwin IT. Agrobacterium-mediated transformation of Nicotiana attenuata, a model ecological expression system. Chemoecology. 2002;12(4):177-83.

46. van Dam NM, Horn M, Mares M, Baldwin IT. Ontogeny constrains systemic protease inhibitor response in Nicotiana attenuata. J Chem Ecol. 2001;27(3):547-68.

47. R Development Core Team: $\mathrm{R}$ : a language and environment for statistical computing. 2.11.0 ed. Vienna, Austria; 2013.

\section{Submit your next manuscript to BioMed Central and take full advantage of:}

- Convenient online submission

- Thorough peer review

- No space constraints or color figure charges

- Immediate publication on acceptance

- Inclusion in PubMed, CAS, Scopus and Google Scholar

- Research which is freely available for redistribution 